\title{
Discrimination between upper versus lower airway components to the rise of total airway resistance measured by Pennock's method after nasal irritant challenge
}

\author{
Zuzana Biringerova $^{1,2}$, Silvia Gavliakova ${ }^{1}$, Eva Hanuskova ${ }^{1}$, Tomas Buday ${ }^{1}$, Jana Plevkova ${ }^{1^{*}}$ \\ ${ }^{1}$ Department of Pathophysiology, Jessenius Faculty of Medicine, Comenius University, Martin, Slovakia \\ ${ }^{2}$ Clinic of Anaesthesiology and Emergency Medicine, Jessenius Faculty of Medicine, Comenius University, Martin, Slovakia

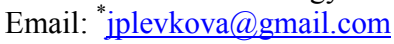

Received 18 June 2013; revised 10 July 2013; accepted 20 July 2013

Copyright (C) 2013 Z. Biringerova et al. This is an open access article distributed under the Creative Commons Attribution License, which permits unrestricted use, distribution, and reproduction in any medium, provided the original work is properly cited.

\section{ABSTRACT}

Terminals of the trigeminal afferents innervating nasal mucosa are called gate keepers, since these fibres detect substances entering the airways. Trigeminal excitation by irritants initiates airway defensive mechanisms, and it is also attributed to the influence of lower airways resistance in a term of nasobronchial reflex. This phenomenon is frequently under debate, because some investigators were unable to confirm its existence. The aim of our study was to determine, whether pharmacological approach could be useful to reach high accuracy and better interpretation of the data obtained by Pennock's method. Pennock's method, which is frequently used to measure airway resistance in vivo (Raw) in fact measures total airway resistance, however, the data are usually interpreted in a terms of bronchomotor response. The upper airway component, which represents approximately $40 \%$ of Raw, is commonly not considered as being important in this method. 30 Dunkin Hartley guinea pigs were exposed to nasal stimuli (TRPA1 agonist-irritant allylisothiocyanate (10 mM, AITC), TRPM8 agonist with cooling potential menthol $\left(10^{-3} \mathrm{M}\right)$ and saline as a control). Raw was measured pre challenge as baseline, after nasal provocation and further, after nasal inhalation of histamine and methacholine $\left(10^{-6}\right.$ $M)$ each. The data showed rise of Raw only after nasal AITC challenge, with further increased responsiveness to histamine and methacholine (5.3 vs 10.18

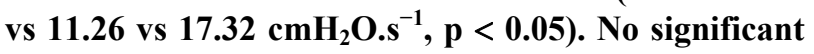
changes were detected after saline, or menthol respectively. Data obtained in further experiment and its analysis showed that pre-treatment with nasal administration of $1 \%$ oxymetazoline but without salbu-

${ }^{*}$ Corresponding author. tamol inhalation prevented the rise of Raw after nasal irritant challenges. Raw after nasal irritant challenges rises rather due to nasal response than due to narrowing of the lower airways.

Keywords: Airways; Resisatnce; In Vivo; Pennock's Method

\section{INTRODUCTION}

Nasal cavity is the first part of the respiratory system which is exposed to the ambient air [1]. Changes of the temperature, humidity and the presence of air born irritants could be potentially dangerous for the respiratory mucosa. Therefore these stimuli could initiate airway protective and defensive reflexes via activation of trigeminal afferents with the properties of nociceptors [2-4].

It is known that trigeminal excitation by different chemicals initiates the sensation of irritation, pain and lacrimation [5]. Furthermore exposure to chemicals such as irritant vapours, air born pollutants or oxidizing substances frequently induces sensation of chest tightness or dyspnoea, sometimes accompanied by wheezing [6]. It is believed that nasal exposure to irritants induces changes in the lower airway resistance named nasobronchial reflex, which is believed to contribute to the mentioned chest sensations. Nasobronchial reflex, as a phenomenon, remains controversial, because the data about its existence share conflicting evidence.

A nasobronchial reflex has been hypothesized to explain the relationship between events occurring in the upper and the lower airways [7]. Sercer suggested the existence of such a reflex after he observed that blowing air into one nostril induced expansion of the ipsilateral thorax [5]. In another study, bronchoconstriction occurred in individuals who had silica dust blown into their 
nose, and in one subject who had previously undergone the unilateral trigeminal nerve resection, bronchoconstriction occurred only when the dust was blown through the neurologically intact side [8]. Also bronchoconstriction provoked by nasal cold dry air, histamine and ammonia challenges had been reported [9-11]. Some investtigators, however, have been unable to confirm nasobronchial reflex [12-14]. Another debate on nasobronchial interaction is that nasal irritation may not be initiating the bronchial response, but it may potentiate a neural reflex initiated in the lower airways [15].

While in the human studies the bronchial narrowing after nasal irritation is measured by spirometry or plethysmography, the results of animal studies are not easy to interpret because of methodological bias. In our research we use the Pennock's method, which measures the airway resistance in vivo, in restrained conscious animals. This method is commonly used to study effects of bronchoactive substances [16,17], however, interpretation of the data obtained by Pennock's method must be considered. There are two main resistors in the respiratory system-upper airway (nasal cavity with the turbinates and the mucosa which can modulate the patency very effectively) and the lower airways with the smooth muscles, which can contract or relax as a response to neural and/or humoral signalling. Pennock's method measures the total airway resistance as it is explained in the original US patent application [18]. We postulated a hypothesis that pharmacological intervention, selectively blocking upper or lower airways component could contribute to the higher accuracy and better interpretation of obtained data. We decided to test the presence of nasobronchial reflex in conscious animal model and relevance of Pennock's method in this observation.

\section{METHODS}

This study was conducted on male Dunking Hartley guinea pigs, obtained from accredited breeding facility (L. Sobota, Městec Králové, Czech Republic). Animals were housed in an approved animal holding facility maintained at controlled room temperature $21^{\circ} \mathrm{C}-22^{\circ} \mathrm{C}$, humidity $60 \%-70 \%$, ventilation, 12-h light-dark cycle and had free access to water and standard animal food.

Animal care was provided and experiments were conducted in agreement with the Animal Welfare Guidelines of the Comenius University and statutes and rules of Slovak Republic legislation. Current study was approved by the decision No: 2999/07-221.

Animals were adapted to laboratory conditions twice. They stayed in plethysmograpic box in order to get familiar with the environment and people responsible for the experimental manipulations. In the plethysmographic box, they had been exposed to the aerosol of buffered saline for 2 minutes that correspond to future ex- perimental procedure and significantly reduces future stress.

\subsection{Nasal Challenges}

Based on the literature data, we decided to use TRPA1 agonist allyl-isothiocyanate (AITC), hot component of horseradish, mustard or wasabi (Sigma). Stock solution of AITC in 1\% DMSO (dimethylsufloxide) was prepared and then further diluted at the day of its use to $10 \mathrm{mM}$ concentrations (maximal effective concentration in conscious guinea pigs for nasal challenge, Brozmanova, personal communication). As a molecule with opposite effects-anti-irritating, calming and cooling-(-) menthol $\left(10^{-3} \mathrm{M}\right)$ TRPM8 agonist has been used. Stock solution prepared in ethanol was further diluted to $10^{-3} \mathrm{M}$. As a negative control to both of these agonists isotonic saline $(0.015 \mathrm{ml})$ warmed up to nearly body temperature $\left(32^{\circ} \mathrm{C}\right)$ was used. All of the solutions were administered bilaterally into the each nostril of experimental animals using a thin plastic flexible catheter (external diameter $1 \mathrm{~mm}$ ) with maximal caution to avoid damage of nasal mucosa (Figure 1).

\subsection{Assessment of Nasal Symptoms}

The symptoms intensity was evaluated by trained persons used a nasal symptom score system which was developed and validated by Brozmanova et al. (2008) matching symptoms intensity to numeric values (maximum 6 and minimum 0 ).

Nasal symptoms score was calculated based on validated scale system. Observed processes were count of sneezes and:

1) nasal discharge: no discharge 0 , mild/moderate 1 , discharge falls in drops from nose 2 .

2 ) eye/conjunctival reaction: no changes 0 , hazy eyes 1 , visible lacrimation 2 .

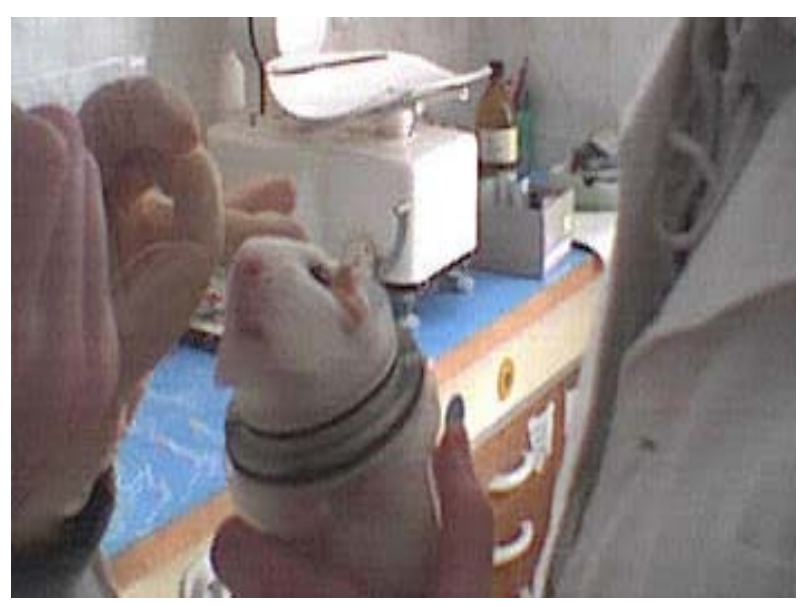

Figure 1. Represents the way of administration of nasal stimuli in restrained conscious guinea pigs. 
3) nasal phenomenon: no crackles 0 , audible crackles 1 , crackles audible from a distance 2 .

\subsection{Measurement of Specific Airway Resistance in Awake Guinea Pigs}

Specific airway resistance (Saw) was measured by a simple non-invasive plethysmographic technique, Pennock's method [18]. Conscious animals were placed in a double chamber body plethysmograph composed of a head chamber and a body chamber (type 855, Hugo Sachs Electronic, Germany). The nasal airflow was measured in the head chamber; the thoracic respiratory airflow was calculated from the pressure changes in the body chamber. Both measurements were made with differential pressure transducers (Simsoft, Martin, Slovakia) and recorded to the PC for Pulmodyn Pennock W software analysis.

In this system, the specific airway resistance is proportional to phase difference between nasal airflow and thoracic respiratory flow [18]. Specific airway resistance was calculated from the phase difference between the thoracic respiratory flow and nasal airflow based on Lissajous loop presentation of the thoracic respiratory flow and nasal airflow on the $\mathrm{X}$ - and Y-axis. Specific airway resistance was measured before the challenges assigned as baseline, then after nasal challenges of AITC, menthol and saline respectively. Finally, resistance was measured also after inhalation of histamine and methacholine aerosols.

In the second round of experiments, resistance was measured before nasal challenges assigned as baseline, then nasal administration of oxymetazoline $1 \%$ and inhalation of salbutamol $(0.1 \mathrm{mg} / \mathrm{ml})$ were used to observe separately upper and lower airway components of the airway resistance after nasal administration of selected agonists AITC $(10 \mathrm{mM})$ and menthol $(10 \mathrm{mM})$.

\subsection{Statistical Analysis of the Data}

Data representing the airway resistance are expressed as mean and standard error of mean. Analysis of variance (ANOVA for repeated measurements) was used to analyze the data. As with any ANOVA, repeated measures ANOVA tests the equality of means. However, repeated measures ANOVA is used when all members of a random sample are measured under a number of different conditions. As the sample is exposed to each condition in turn, the measurement of the dependent variable is repeated. Using a standard ANOVA in this case is not appropriate because it fails to model the correlation between the repeated measures: the data violate the ANOVA assumption of independence. $\mathrm{p}<0.05$ was considered as being statistically significant.

\section{RESULTS}

\subsection{Nasal Symptoms Score Induced by Selected Nasal Challenges}

Nasal administration of saline warmed up to body temperature $(0.015 \mathrm{ml}$, bilateral challenge using thin plastic catheter) induced only a sneezing reflex, 2 - 3 sneezes per nostril in average, very likely because of mechanical irritation of the nerves on the turbinate mucosa. No other symptoms were observed. Administration of $0.015 \mathrm{ml}$ of $l$-menthol isomer $\left(10^{-3} \mathrm{M}\right.$, bilateral challenge) induced similar sneezing response, and additionally, 4/15 guinea pigs rubbed their noses by the front limb, this reaction was transient, restricted to the interval immediately after the administration. Nasal administration of an airway irritant allyl-isothiocyanate (AITC) induced massive sneezing response, significantly higher than that observed after nasal saline or menthol challenges $(3 \pm 1$ vs $10 \pm 4$ sneezes per observed period of 15 minutes, $p<$ $0.05)$. Except robust activation of sneezing reflex, animals developed rhinorrhoea with nasal discharge, they rubbed their noses by the front limb, and they also developed nasal acoustic phenomena (crackles) which are signs of nasal obstruction with secretion in the nasal passages.

These reactions were taken as an evidence for robust activation of nasal trigeminal afferents, particularly nociceptors. Menthol in this concentration was expected to stimulate distinct population of neurons, (TRPM8+/ TRPV1-population of trigeminal afferents), therefore no nocifensive reactions were observed.

\subsection{Specific Airway Resistance Measured by Pennock's Method after Nasal Challenges}

Specific resistance was measured before nasal challenges (assigned as basal), then after nasal challenges of saline $(0.015 \mathrm{ml}), l$-menthol and AITC (bilateral challenges) (assigned as after nasal challenge) and finally, animals in plethysmographic box were exposed to histamine aerosol, and methacholine aerosol to observe airway reactivity to most frequently used bronchoactive substances - more in methods section). Figure 2 represents the change of specific airway resistance after nasal AITC, saline and $l$-menthol challenges.

The specific resistance measured by Pennock's method was not significantly influenced after nasal challenges with saline and menthol. The rise of resistance was spotted after nasal AITC challenge, suggesting for nasobronchial reflex. Resistance rose even higher after inhalation of histamine and methacholine respectively, what was expected (5.3 vs 10.18 vs 11.26 vs 17.32 $\left.\mathrm{cmH}_{2} \mathrm{O} \cdot \mathrm{s}^{-1}, \mathrm{p}<0.05\right)$. Animals with nasal saline and $l$-menthol challenges responded to histamine and methacholine less significantly than animals with nasal AITC 


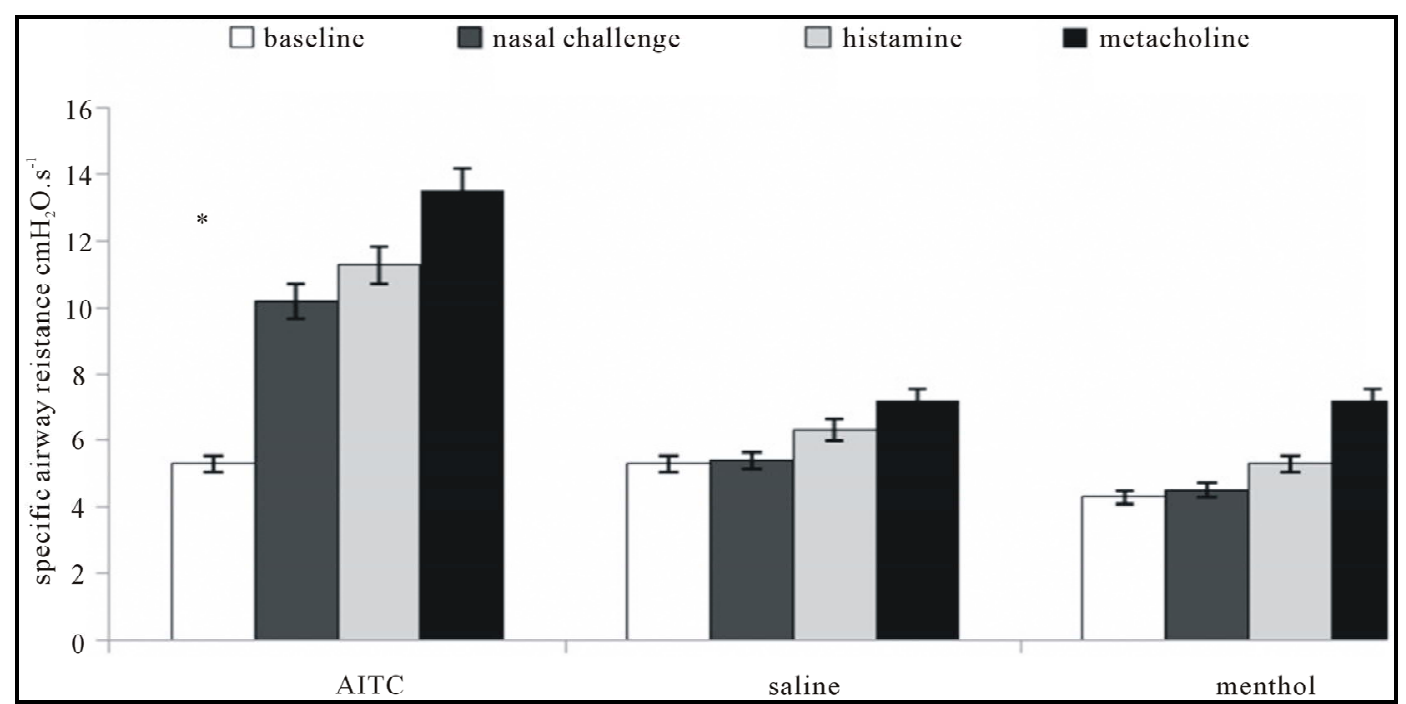

Figure 2. Represents the rise of the airway resistance after nasal administration of an irritant AITC $10 \mathrm{mM}$. Inhalation of histamine and methacholine provoked significant increase when comparing to baseline, however not significantly different with the value obtained after nasal AITC challenge. Additional rise of the resistance after histamine and methacholine could be attributed to the bronchomotor response, as the upper airway component is already involved. Nasal administration of saline and menthol did not provoke the rise of the airway resistance just by themselves. Further inhalation of histamine and methacholine provoked the rise of airway resistance probably by the combined upper and lower airway influencing mechanisms. ${ }^{*} \mathrm{p}<0.05$.

(data in saline and menthol lines were not significantly changed). This is a circumstantial evidence confirming increased responsiveness of the lower airway once the nasal afferents are robustly activated.

\subsection{Specific Airway Resistance Measured by Pennock's Method after Nasal AITC - Effects of Nasal Decongestant versus $\beta_{2}$ Mimetics Pre-Treatment}

In further studies we focused on the effects provoked by nasal irritant AITC after selective blocks of nasal reactivity by nasal pre-treatment with $1 \%$ oxymetazoline $\left(\alpha_{1}\right.$ agonist and potent decongestant), which blocks nasal vascular reactivity and salbutamol- $\beta_{2}$ agonist, which has bronchodilatatory effects.

Figure 3 documents the decrease of airway resistance measured by Pennock's method from baseline $(5.3 \pm 0.25$ to $\left.3.7 \pm 0.3 \mathrm{cmH}_{2} \mathrm{O} \cdot \mathrm{s}^{-1}, \mathrm{p}<0.05\right)$ after the nasal cavity was pre-treated with $1 \%$ oxymetazoline. Nasal administration of AITC $10 \mathrm{mM}$ to the nose pre-treated with oxymetazoline $1 \%$ did not induce significant rise of the airway resistance. Further inhalation of salbutamoll did not modulate the airway resistance in this model, confirming the role of the upper rather than a lower airway component to the total airway resistance after nasal challenge of airway irritants.

\section{DISCUSSION}

In our experimental setup, Pennock's method was used to measure changes of the airway resistance in vivo in restrained conscious guinea pigs after stimulation of trigeminal afferents by the airway irritant AITC. Nasal administration of an irritant AITC induced noticeable nasal symptoms, thus providing an indirect evidence of robust activation of nasal sensory nerves with somatomotoric, vascular and glandular response to this activation. It is believed that irritation of nasal afferents induces changes in the lower airway lumen, leading to the narrowing of the bronchi through bronchoconstriction (nasobronchial reflex).

Nasal administration of AITC induced the rise of the airway resistance measured by Pennock's method. Nasal administration of menthol and saline did not induce the rise of airway resistance at all (Figure 2). This result could lead to an interpretation, that nasal challenge with irritants induced rise of the specific airway resistance, which could be furthermore interpreted as an evidence of nasobronchial reflex. However, pre-treatment with salbutamol and nasal decongestant oxymetazoline clearly showed that AITC induced rather nasal vascular congestion and mucosal swelling than bronchoconstriction. If there was a bronchoconstriction present, this would lead to decrease of the resistance after inhalation of salbutamol in the second round of experiments (Figure 3).

This result is highly indicative of inaccuracy of the results obtained by Pennock's method as they do not count the upper airway component to the airway resistance, which is approximately $40 \%-50 \%$ [5]. Measurement of the airway resistance in restrained animals is 


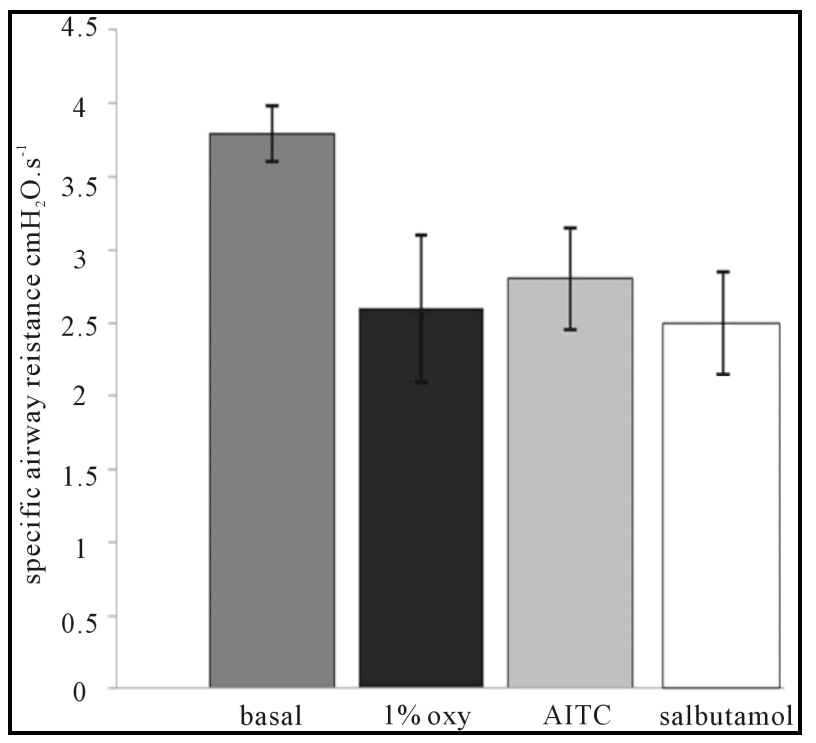

Figure 3. Documents the role of the vascular component of the upper airway resistance after $1 \%$ oxymetazoline pre-treatment with no further rise of the airway resistance after intranasal administration of $10 \mathrm{mM}$ AITC. Inhalation of salbutamol 0.1 $\mathrm{mg} / \mathrm{ml}$ did not lead to any additional changes of the airway resistance.

a very useful tool in the pharmacology and physiology measurements. Non-invasive airway mechanics provide information about airway function comparable to more invasive approaches, while retaining the inherent advantages of being more physiological and suitable for longitudinal follow up [16,17]. However, we do believe that upper airway component must be taken into consideration. Selective block of the nasal reactivity by nasal decongestants could bring more accuracy to the observations, because the data for lower airway component (smooth muscle) will become more reliable.

In double chamber plethysmograph, or whole body restrained measurement of the airway resistance, animals are exposed to histamine or methacholine aerosol respectively to observe the airway responsiveness to these most frequently used mediators. However, histamine receptors are expressed also on the nasal structures, which leads to mucosal swelling and discharge on exposure. Similarly, methacholine considerably activates glands in nasal airway mucosa through cholinergic signalling [5].

Although the guinea pigs are obligatory nasal breathers, they inhale these mediators through the nose, thereby inducing effects in upper and lower airways as well. Interpretation of such a result as a measure of bronchoconstriction would be very inaccurate. Logically, if an animal is inhaling histamine or methacholine through intact airways, this would lead to decreasing patency of nasal passages (congestion, mucus output), but also decreasing patency of the lower airways via bronchoconstriction. The most important component contributing to the nasal airway resistance is the vascular reaction-vasodilatation and venous sinuses engorgement, therefore, administration of nasal decongestants prior to the measurement of the bronchomotor response will block or limit the upper airway response, thereby referring more to lower airway response.

Oxymetazoline is a topically applied nasal decongesttant that has a vasoconstrictive effect on the nasal mucosal blood vessels, thereby, rapidly relieving symptoms of nasal congestion. It acts as $\alpha$-adrenergic agonist on receptors of the vascular smooth muscle, constricting the venous sinusoids within the nasal mucosa. This reduces blood flow through the nasal mucosa resulting in decreased nasal oedema and a reduced sensation of nasal congestion. In common clinical doses and local administration, it is an effective decongestant without systemic effects $[19,20]$. These can be observed only after accidental ingestion of $1-2 \mathrm{ml}$ of decongestants (cardiovascular and metabolic effects and urine retention in subjects with hyperplastic prostatic gland mediated by $\alpha$-adrenergic receptors).

To study the upper versus lower airway contribution to the airway resistance, AITC and menthol were used as interesting stimuli.

AITC is an agonist of TRPA1 channel, abundantly expressed on trigeminal nerves, and this channel based on its neurophysiological properties, activation profile and responsiveness to majority of air born pollutants, oxidizing substances and products of oxidative stress is believed to play a role of an airway irritant receptor [21]. Therefore, we decided to use a potent and robust activetor of this channel, leading to noticeable symptoms and signs of nasal irritation. As an opposite mediator, we have chosen menthol, which is known for its calming, cooling, and anti-irritating effects. It is an agonist of TRPM8 channels, and its administration induces completely opposite sensations when applied to humans. For the upper airways, it is frequently used to relieve the nasal symptoms, particularly the nasal obstruction, as it induced feeling of better nasal airflow due to stimulation of cold/flow trigeminal receptors [22-24]. As a negative control to each of them, we decided to use saline warmed up to body temperature, as it is known that thermosensors are also abundantly expressed on nasal trigeminal afferents. Temperature and osmotic activity equal to the mucosal bilaminar cover makes it perfect to control challenge medium.

Nasal administration of AITC in our experiments increased nasal mucus output and swelling via activation of TRPA1 channel, which was manifested as acoustic nasal phenomena and laboured breathing. Mentioned effects were blocked by nasal pre-treatment with $1 \%$ oxymetazoline, thus suggesting for vascular component. Specific resistance measured after administration of this 
potent decongestant dropped significantly from the baseline, providing evidence that nasal mucosal congestion contributes significantly to the airway resistance in normal conditions, and this could even rise if the upper airways are exposed to the irritants.

In vivo measurement of the airway resistance by Pennock's method can bring very useful data about the airway mechanics; however, upper and lower airway components to the total airway resistance must be taken into consideration. Selective pharmacological block of the nasal vascular response by decongestants can cut off positive false results which are obtained in fact by the reaction of the upper airways.

\section{ACKNOWLEDGEMENTS}

Study was supported by VEGA no. 1/0031/11 and Centre of experimental and clinical respirology II co-financed from EU sources. The authors acknowledge Assoc. Prof. M. Sutovska and Dr. M. Kocmalova from Department of Pharmacology JFM CU for their outstanding help with the measurement of the airway resistance.

\section{REFERENCES}

[1] Panneton, W.M., Hsu, H. and Gan, Q. (2010) Distinct central representations for sensory fibers innervating either the conjunctiva or cornea of the rat. Experimental Eye Research, 90, 388-396. doi:10.1016/j.exer.2009.11.018

[2] Wallois, F., Macron, J.M., Jounieaux, V. and Duron, B. (1991) Trigeminal nasal receptors related to respiration and to various stimuli in cats. Respiration Physiology, 85, 111-125. doi:10.1016/0034-5687(91)90010-G

[3] Sekizawa, S. and Tsubone, H. (1994) Nasal receptors responding to noxious chemical irritants. Respiration Physiology, 96, 37-48. doi:10.1016/0034-5687(94)90104-X

[4] Taylor-Clark, T.E., Kollarik, M., MacGlashan Jr., D.W. and Undem, B.J. (2005) Nasal sensory nerve populations responding to histamine and capsaicin. The Journal of Allergy and Clinical Immunology, 116, 1282-1288. doi:10.1016/j.jaci.2005.08.043

[5] Baraniuk, J.N. and Merck, S.J. (2008) Nasal reflexes: Implications for exercise, breathing and sex. Current Allergy \& Asthma Reports, 8, 147-153. doi:10.1007/s11882-008-0025-7

[6] Taylor-Clark, T.E. (2008) Insights into the mechanisms of histamine-induced inflammation in the nasal mucosa. Pulmonary Pharmacology \& Therapeutics, 21, 455-460. doi:10.1016/j.pupt.2007.08.002

[7] Sahin-Yilmaz, A. and Naclerio, R.M. (2011) Anatomy and physiology of the upper airway. Proceedings of American Thoracic Society, 8, 31-39. doi:10.1513/pats.201007-050RN

[8] Kaufman, J., Chen, J.C. and Wright, G.W. (1970) The effect of trigeminal resection on reflex bronchoconstriction after nasal and nasopharyngeal irritation in man. The American Review of Respiratory Disease, 101, 768-769.

[9] Togias, A. (1999) Mechanisms of nose lung interaction.
Allergy, 54, 94-105.

[10] Johansson, A., Bende, M., Milquist, E. and Bake, B. (2000) Nasobronchial relationship after cold air provocation. Respiratory Medicine, 94, 1119-1122. doi:10.1053/rmed.2000.0924

[11] Fontanari, P., Burnet, H., Zattara-Hartmann, M.C. and Jammes, Y. (1996) Changes in airway resistance induced by nasal inhalation of cold dry, dry, or moist air in normal individuals. Journal of Applied Physiology, 81, 1739-1743.

[12] Levi, L.R., Tyler, G.R., Olson, L.G. and Saunders, N.A. (1990) Lack of airway response to nasal iritation in normal and asthmatic subjects. Australian and New Zealand Journal of Medicine, 20, 578-582.

[13] Little, N.T., Carlisle, C.C., Millman, R.P. and Braman, S.S. (1990) Changes in airway resistance following nasal provocation. The American Review of Respiratory Disease, 141, 580-583. doi:10.1164/ajrccm/141.3.580

[14] Cole, P. (1998) Physiology of the nose and paranasal sinuses. Clinical Reviews in Allergy \& Immunology, 16, 33-51. doi:10.1007/BF02739327

[15] Plevkova, J., Kollarik, M., Brozmanova, M., Revallo, M., Varechova, S. and Tatar, M. (2004) Modulation of experimentally-induced cough by stimulation of nasal mucosa in cats and guinea pigs. Respiratory Physiology \& Neurobiology, 142, 225-235.

doi:10.1016/j.resp.2004.06.006

[16] Tohda, Y., Muraki, M., Iwanaga, T., Haraguchi, R., Fukuoka, M. and Nakajima, S. (2000) Dual-phase response model for bronchial asthma. Allergy and Asthma Proceedings, 21, 79-84. doi:10.2500/108854100778250851

[17] Lofgren, J.L., Mazan, M.R., Ingenito, E.P., Lascola, K., Seavey, M., Walsh, A. and Hoffman, A.M. (2006) Restrained whole body plethysmography for measure of strain-specific and allergen induced airway responsiveness in conscious mice. Journal of Applied Physiology, 101, 1495-1505. doi:10.1152/japplphysiol.00464.2006

[18] Pennock, B.E., Cox, C.P., Rogers, R.M., Cain, W.A. and Wells, J.H. (1979) A non-invasive technique for measurement of changes in specific airway resistance. Journal of Applied Physiology, 46, 399-406.

[19] Agrawal, A. and Ram, A. (2007) Commentary on "restrained whole body plethysmography for measure of strain-specific and allergen-induced airway responsiveness in conscious mice". Journal of Applied Physiology, 102, 2412-2413. doi:10.1152/japplphysiol.00134.2007

[20] Bickford, L., Shakib, S. and Taverner, D. (1999) The nasal airways response in normal subjects to oxymetazoline spray: Randomized double-blind placebo-controlled trial. British Journal of Clinical Pharmacology, 48, 53-56. doi:10.1046/j.1365-2125.1999.00972.x

[21] Gerhold, K.A. and Bautista, D.M. (2008) TRPA1: Irritant receptor of the airways. The Journal of Physiology, 586, 3303 .

[22] Sekizawa, S., Tsubone, H., Kuwahara, M. and Sugano, S. (1996) Nasal receptors responding to cold and 1-menthol airflow in the guinea pig. Respiration Physiology, 103, 211-219. doi:10.1016/0034-5687(95)00091-7

[23] Orani, G.P., Anderson, J.W., Sant'Ambrogio, G. and 
Sant'Ambrogio, F.B. (1991) Upper airway cooling and 1 -menthol reduce ventilation in the guinea pig. Journal of Applied Physiology, 70, 2080-2086.
[24] Eccles, R. (1994) Menthol and related cooling compounds. The Journal of Pharmacy and Pharmacology, 46, 618630. 